\title{
Surgical Masculinization of the Breast: Clinical Classification and Surgical Procedures
}

\author{
Lázaro Cárdenas-Camarena $^{1}\left(\mathbb{D} \cdot\right.$ Carlos Dorado $^{1} \cdot$ Maria Teresa Guerrero $^{1}$. \\ Rosa Nava ${ }^{1}$
}

Received: 24 June 2017 / Accepted: 13 July 2017 / Published online: 21 July 2017

(C) Springer Science+Business Media, LLC and International Society of Aesthetic Plastic Surgery 2017

Level of Evidence $V$ This journal requires that authors assign a level of evidence to each article. For a full description of these Evidence-Based Medicine ratings, please refer to the Table of Contents or the online Instructions to Authors www.springer.com/00266

We appreciate the comments of Dr. Innocenti et al. about our work of masculinization of the mammary gland. The concepts that Dr. Innocenti handles on the importance of BMI for better results and higher patient satisfaction are valid. Obviously, the expectations of each patient will be very different depending on their physical preoperative characteristics. Patients with athletic bodies and low body mass index always demand and expect a more perfect result than those who are overweight. This type of behavior and expectations are seen in multiple cosmetic surgical procedures. Patients with a higher body mass index, who become overweight and even obese, are more tolerant in the results that can be obtained, not because the results are less adequate, but because small procedures in them produce great changes, surpassing their physical and psychological expectations. However, we also consider that the degree of satisfaction of a surgery depends primarily on an appropriate patient medical relationship. Being clear and specific with what we can offer and the results that we can obtain, the patient will generally be satisfied. In case of not accepting the result offered, always the recommendation is not to be operated.

Fortunately, in the patients of this study we achieved a high index of complete satisfaction, close to $90 \%$. This satisfaction is high because the correction of gynecomastia or the masculinization of the mammary gland is one of the surgical procedures in which the elimination of the mammary tissue produces the changes that the patient expects, although in many of them the scars are not as good as we always expect.

\section{Compliance with Ethical Standards}

Conflict of interest The authors have no conflicts of interest to declare in relation to the content of this article.

Lázaro Cárdenas-Camarena

drlazaro@drlazarocardenas.com

1 Innovare Cirugia Plástica Especializada, Av. Verona 7412

Villa Verona, Zapopan, Jalisco, Mexico 